\title{
Do thermal effects cause the propulsion of bulk graphene material?
}

To the Editor - In a recent article, Zhang et al. ${ }^{1}$ observed the direct propulsion of a bulk graphene sponge when exposed to laser light. They attributed this to the momentum of the light-induced ejected electrons. However, the force provided by the ejected electrons is about $2.7 \times 10^{-11} \mathrm{~N}$ (supposing that the average current and the kinetic energy of the ejected electrons are $9 \times 10^{-7} \mathrm{~A}$ and $70 \mathrm{eV}$, respectively), which is far smaller than the gravitational force of a $0.86 \mathrm{mg}$ graphene sponge. From our knowledge of rarefied gas dynamics, the horizontal, vertical and rotational motion of the laser-illuminated graphene sponge could be due to the radiometric force.

The effect of the radiometric force is most commonly seen in the Crookes radiometer - an airtight glass bulb in which there is a partial vacuum. Inside the bulb is a set of thin vanes mounted on a spindle. Each vane has a blackened side and a silvered side. The vanes rotate when exposed to light, with the silvered surfaces leading the motion. The reason for this rotation was much debated following the invention of the device, but in 1879 the currently accepted explanation for the rotation was proposed by Osborne Reynolds ${ }^{2}$, who attributed it to thermal transpiration. In brief, when a gas molecule collides with a surface, it is diffusely reflected; for a surface with a temperature gradient, a molecule from the hotter side loses more momentum than a molecule from the colder side. This is the source of a 'radiometric' force that is applied on the surface in the direction from the hotter side to the colder side.

The graphene sponge propulsion experiment was conducted in a glass tube that contained air at a pressure of about $0.1 \mathrm{~Pa}$. The mean-free path of the air molecules in this tube is $65 \mathrm{~mm}$, which is much larger than the diameter of the graphene sponge. So collisions between air molecules and the solid surfaces of both the graphene sponge and the tube outnumber collisions between air molecules. According to the kinetic theory of gases $^{3}$, the radiometric force is:

$$
\begin{gathered}
F=\frac{P}{2} A\left(\sqrt{\frac{\sigma_{\mathrm{M}} T_{\mathrm{h}}+\left(1-\sigma_{\mathrm{M}}\right) T_{\infty}}{T_{\infty}}}-\right. \\
\left.\sqrt{\frac{\sigma_{\mathrm{M}} T_{\mathrm{c}}+\left(1-\sigma_{\mathrm{M}}\right) T_{\infty}}{T_{\infty}}}\right)
\end{gathered}
$$

where $P$ is the air pressure before laser illumination, $A$ is the cross-sectional area of the graphene sponge, $T_{\mathrm{h}}$ and $T_{\mathrm{c}}$ are the temperatures at the bottom and the top of the illuminated graphene sponge, $T_{\infty}$ is the temperature of the glass tube, and $\sigma_{\mathrm{M}}$ is the tangential momentum accommodation coefficient of the surface. Assuming $\sigma_{\mathrm{M}}=1$, and $T_{\mathrm{c}}=T_{\infty}=300 \mathrm{~K}$, the radiometric force is larger than the gravitational force of a $0.86 \mathrm{mg}$ graphene sponge when $T_{\mathrm{h}}=3,000 \mathrm{~K}$.

Such a high temperature difference $(2,700 \mathrm{~K})$ between the bottom and the top of the graphene sponge may be possible, as Zhang et al. estimated that with a laser pulse width of $2 \mathrm{~ms}$ and a laser power of $3 \mathrm{~W}$ the average temperature increase of the graphene sponge is $822 \mathrm{~K}$ if all the laser energy is converted to thermal energy. Since the absorbance of a single layer of graphene is $2.3 \%$, the temperature at the bottom of the graphene sponge will be over 7,000 K if radiation and heat conduction are ignored. Even if we consider heat conduction for a graphene sponge of length $11 \mathrm{~mm}$, density $1 \mathrm{mg} \mathrm{ml}^{-1}$, and a conductivity of $0.5 \mathrm{~S} \mathrm{~m}^{-1}$, a uniform temperature will only be reached after a characteristic time of $17 \mathrm{~ms}$. This time interval is comparable to the time for the establishment of the radiometric force (supposing that the distance between the bottom of the glass tube and the graphene sponge is $10 \mathrm{~cm}$, and the characteristic time for the gas molecules travelling between the glass tube and the graphene sponge is about $0.5 \mathrm{~ms}$ ). The time required for the establishment of the radiometric force is dozens of this characteristic time, which is about $10-20 \mathrm{~ms}$.

If the radiometric force is the cause of the motion of the graphene sponge, then it is impossible for the solar sail proposed by Zhang et al. to be driven directly by sunlight with propulsion orders of magnitude greater than the radiation pressure, since the radiometric force vanishes in the vacuum of deep space.

Finally, it is interesting to note that Sir William Crookes invented his radiometer in 1873 as a spin-off from his chemistry research. He was weighing samples in a partially evacuated chamber to reduce the effect of air currents, but noticed his measurements were disturbed when sunlight shone on the balance. Zhang et al. also placed their graphene sponge into a (near) vacuum to avoid the probable interference of air - so history may have repeated itself.

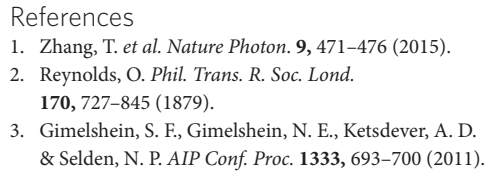

Lei Wu ${ }^{1 \star}$, Yonghao Zhang ${ }^{1}$, Yian Lei $^{2}$ and Jason M. Reese ${ }^{3}$

'James Weir Fluids Laboratory, Department of Mechanical and Aerospace Engineering, University of Strathclyde, Glasgow G1 1XJ, UK. ${ }^{2}$ School of Physics, Peking University, Beijing 100871, China. ${ }^{3}$ School of Engineering, University of Edinburgh, Edinburgh EH9 3FB, UK.

*e-mail: lei.wu.100@strath.ac.uk

\section{Reply to 'Do thermal effects cause the propulsion of bulk graphene material?'}

\begin{abstract}
Zhang et al. reply - In our work ${ }^{1}$, we experimentally synthesized a bulk 3D crosslinked graphene material that exhibits the intrinsic properties of individual graphene sheets. This includes the unique Dirac-type electronic band structure of
\end{abstract}

graphene, which is the origin of many of its remarkable electronic and optoelectronic properties (partially demonstrated in this work $^{1}$ ), as well as its mechanical properties (shown in our earlier paper ${ }^{2}$ ). In our view, the fact that the intrinsic properties of individual graphene sheets are retained in the bulk state is probably the most important implication of our work because "it has been a great challenge to realize the intrinsic properties of single-layer graphene in the bulk state, because stacking 